\title{
Integrated Approach for Matching Statistical Shape Models with Intra-operative 2D and 3D Data
}

\author{
M. Fleute ${ }^{1}$, S. Lavallée ${ }^{1}$, and L. Desbat ${ }^{2}$ \\ 1 PRAXIM, 4 Av. Obiou, 38700 La Tronche, France \\ 2 TIMC Laboratory, University Joseph Fourier, Grenoble, France
}

\begin{abstract}
This paper presents an approach to the problem of intraoperative reconstruction of $3 \mathrm{D}$ anatomical surfaces. The method is based on the integration of intra-operatively available shape and image data of different dimensionality such as $3 \mathrm{D}$ scattered point data, 2.5D ultra sound data, X-ray images etc. by matching them to a statistical shape model, thus providing the surgeon with a complete surface representation of the object of interest. Previous papers of the authors describe the matching of either 3D or 2D data to a statistical model and clinical applications. The here presented work combines former published ideas with a new approach for the complex task of shape analysis required for the computation of the statistical model, thus providing a generic approach for intra-operative surface reconstruction based on statistical models. The method for shape extraction/analysis is based on a generic model of the object and is used to segment training shapes and to establish point to point correspondence simultaneously in a set of CT images. Reconstruction experiments are performed on a statistical model of lumbar vertebrae. Results are provided for 3D/3D, 2D/3D and hybrid matching with simulated data and for $3 \mathrm{D} / 2 \mathrm{D}$ matching for a cadaveric spine.
\end{abstract}

\section{Introduction}

One key issue in CAS systems is the availability of patient specific 3D models of the anatomical structures on which the surgery is performed. For many applications the computation of detailed (and precise) 3D gray scale information based on pre-operative imaging is not mandatory, i.e. reconstruction of the organ shape is sufficient. Therefore it is desirable to be able to infer 3D-information from intra-operative data only to facilitate the navigation within the patient and thus allowing to abandon CT data acquisition (pre- or intra-operatively) at least for many standard surgical applications.

In many existing CAS-systems optical localizers or laser scanners are used to acquire scattered point data on patients' bone surfaces in order to register physical space with pre-operative images. Furthermore X-ray images are the dominating image modality in the operating room. In previous papers authors presented approaches aiming at reconstructing 3D anatomical surfaces for multiple purposes such as surgical planning and visualization relying on such intraoperative data only [FL98FL99]. In order to infer the complete 3D shape of

T. Dohi and R. Kikinis (Eds.): MICCAI 2002, LNCS 2489, pp. 364-372 2002.

(C) Springer-Verlag Berlin Heidelberg 2002 
the object of interest it is necessary to incorporate a priori knowledge into the reconstruction algorithm. The statistical shape model introduced by Cootes and Taylor describes the average shape and characteristic shape variation of a set of training samples that are defined by a corresponding set of boundary points. Along with its simple representation, the capability of dealing with arbitrary shape topologies favours the use of this model. The shape reconstruction is carried out by fitting the data to a statistical shape model based on a generalization of the Iterative closest point algorithm (ICP) BM92.

Two-dimensional statistical shape models have been in use for years now and are well established. Two crucial problems have prevented them so far from being widely used in the $3 \mathrm{D}$ case. First, the necessary segmentation of a sufficient high number of training shapes for the statistical analysis, usually available in the form of a CT exam, is a cumbersome and tedious task using today's available manual or semi-automatic segmentation tools. Second, it is necessary to establish point to point correspondence between all training shapes. This is a nontrivial task and becomes manually infeasible. Few known automatic methods address this problem and rely on already segmented images. The present work proposes a new approach to address both above mentioned problems simultaneously. A generic model of the object is used to segment the training shapes in CT images and to establish point to point correspondence (semi-landmark positioning). A volumetric coarse to fine deformation method based on free form deformations is used to match the generic model to the image data. It is further shown in this work that the combination of both data sources - X-ray images and 3D point data - allowing to perform hybrid registration with the statistical shape model, might be a very interesting option for certain applications. Figure 1 shows a flow diagram of the chosen global approach whose different components are presented in the following sections.

\section{Automatic Shape Extraction from CT Images for Statistical Model Computation}

Prior to establishing correspondence between the training shapes which is required for the statistical analysis, the training shapes must be segmented from the raw $3 \mathrm{D}$ data (CT-data). This segmentation is usually achieved in a slice by slice fashion, which is rather circumstantial, time-consuming and labor-intensive. To overcome these inconveniences the template matching and the data segmentation can be coupled together, i.e. performed simultaneously.

Based on a general shape template a representation of the external external surface is inferred from the model to the data providing a dense set of corresponding points between the template and the data, which can be used for the subsequent statistical analysis. It is worth to note that the matching will be based only on object boundaries, in contrast to the work in ea01a for instance, where the gray level information of the whole volume is used to drive the deformation. However the resulting deformation will be volumetric thus allowing for automatic inference of other structures embedded in the volume. In our 


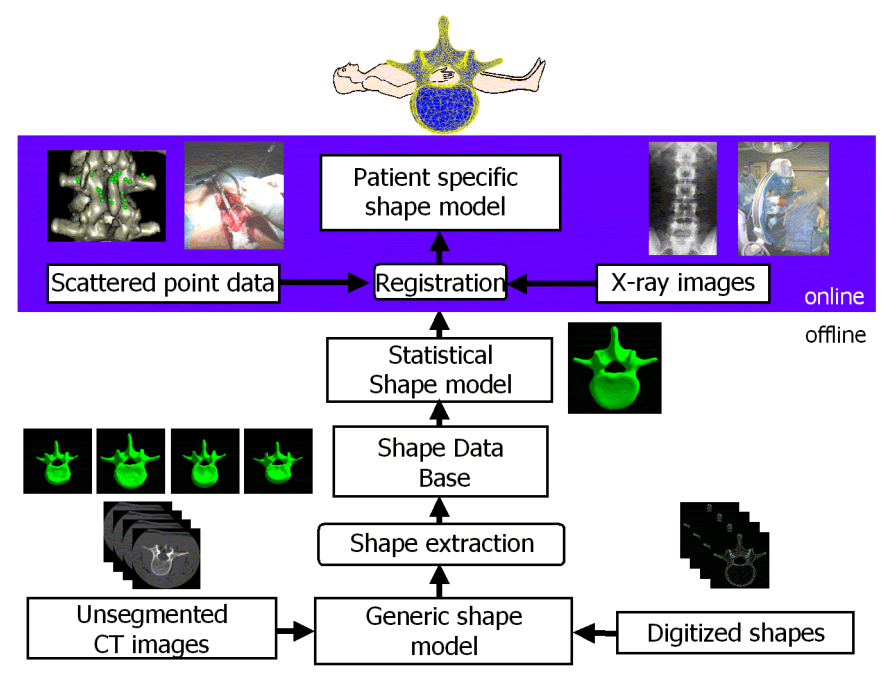

Fig. 1. Flow diagram for Shape Recovery by Nonrigid Registration of a Statistical Shape Model with intra-operative data. The statistical shape model is computed offline and can be matched intra-operatively with $2 \mathrm{D}$ or $3 \mathrm{D}$ data. It is also possible to use both type of data simultaneously (hybrid matching)

approach we use a volumetric coarse to fine registration method based on FreeForm Deformations [SP86] in order to match the generic model to the image data.

The method performs a least square minimization of the distances between model boundary points and matched feature points. The deformation defined by the computed displacement field is described as a warping of the space containing the surface model, based on 3D tensor-product deformation splines. For increased efficiency, the Free-Form Deformations are applied in a multi-resolution framework. The result is a rapid and efficient registration algorithm which does not require the prior segmentation (manual or automatic) of features in the data image, and which can work on arbitrarily shaped surfaces. The proposed method is conceptually following [SL96] but introducing important improvements and extensions sharing ideas from ea99 Pic97 ea00. The problem can be formulated as a minimization of a cost function

$$
E(\mathbf{p})=\sum_{i=1}^{N}\left[\operatorname{dist}\left(P_{i}, T_{\mathbf{p}}\left(M_{i}\right)\right)\right]^{2}+\mathcal{R}(\mathbf{p}),
$$

where dist is the Euclidean distance between a model point $M_{i}$ and its corresponding data point $P_{i}$ in the gray level image. $T$ is a suitable deformation function depending on the parameter vector $\mathbf{p}$ and where $\mathcal{R}$ defines a regularization term which is applied to $T$ in order to smooth the deformation. 

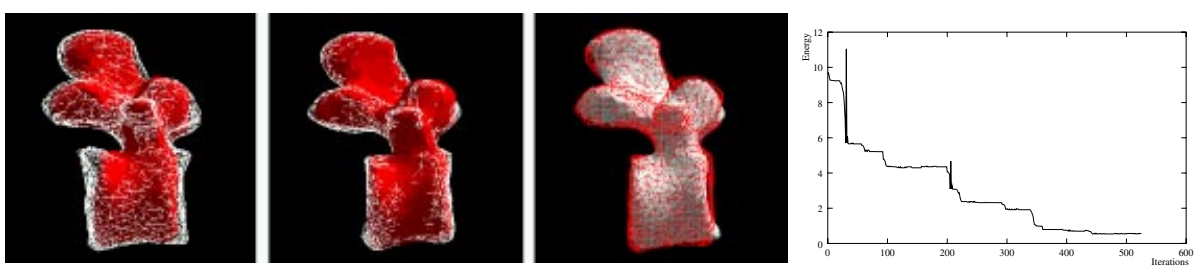

Fig. 2. Left: The influence of different bone thresholds on the resulting model. The mesh represents the registered model, the solid model represents the manually segmented CT model for comparison : (a) too low threshold , (b) optimal threshold, (c) too high threshold. Right: Evolution of the energy during function minimization

The generic model is represented by a triangle mesh. The original mesh obtained by applying the marching cube algorithm to a manually segmented vertebra CT-exam is decimated and smoothed to avoid overfitting.

Two methods have been investigated to attract the model boundary to the object contours in the gray-level image. The first method is based on the bone threshold in the gray level image. Experiments performed on CT images of the vertebral column have shown that good segmentation results can be obtained except for regions where two neighboring vertebrae are articulating and thus the bone gaps are too narrow. Figure 2 shows the effect of varying the bone threshold on the final registered model. In (a) the chosen threshold is too low, in (b) it is optimal (found experimentally) and in (c) it is too high. A second approach based on a statistical gray-level model for each model vertex leads to similar results.

The optimization of $E(\mathbf{p})$ is performed using a conjugate gradient algorithm PFTV92] and a multi resolution representation of $T$ in order to smooth the solution and to speed up the minimization. Initial registration is performed by manual alignment. Figure 2 illustrates the evolution of the energy as a function of the number of iterations. The steps correspond to resolution changes of the deformation grid.

A statistical model of lumbar vertebrae has been computed based on the statistical analysis of 30 vertebrae (L1-L4). Fig. 4. left shows the effect of applying \pm 2 standard deviations of the first two modes of the obtained model to the mean shape. Fig. 4, right shows the captured variability of the statistical vertebra model as a function of the first $\mathrm{n}$ eigenmodes.

\section{Experiments and Results}

3D matching. In a first experiment about 450 points were collected on the dorsal surface of a lumbar vertebra model in an area accessible during spine surgery by sliding a virtual pointer over the model surface. The computed statistical model was then matched to the scattered data. Figure 3 a) shows the mean shape rigidly matched to vertebrae. Figure 3 b) shows the deformed model after applying the nonrigid matching algorithm. One may observe that the overall fit 

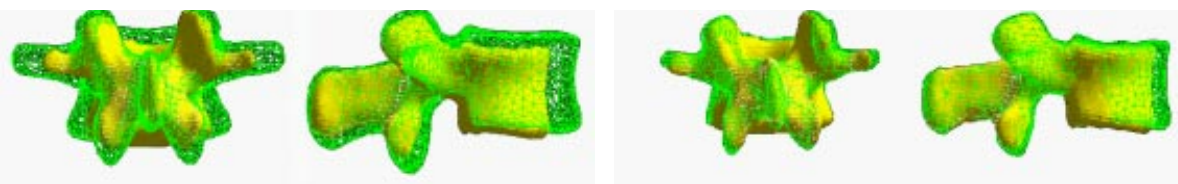

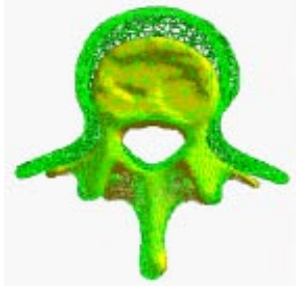

a)
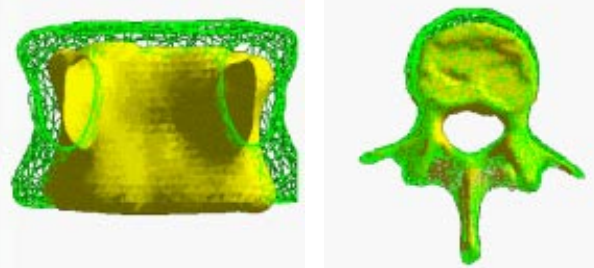

b)

Fig. 3. 3D/3D registration. a) after rigid registration, (b) after nonrigid registration
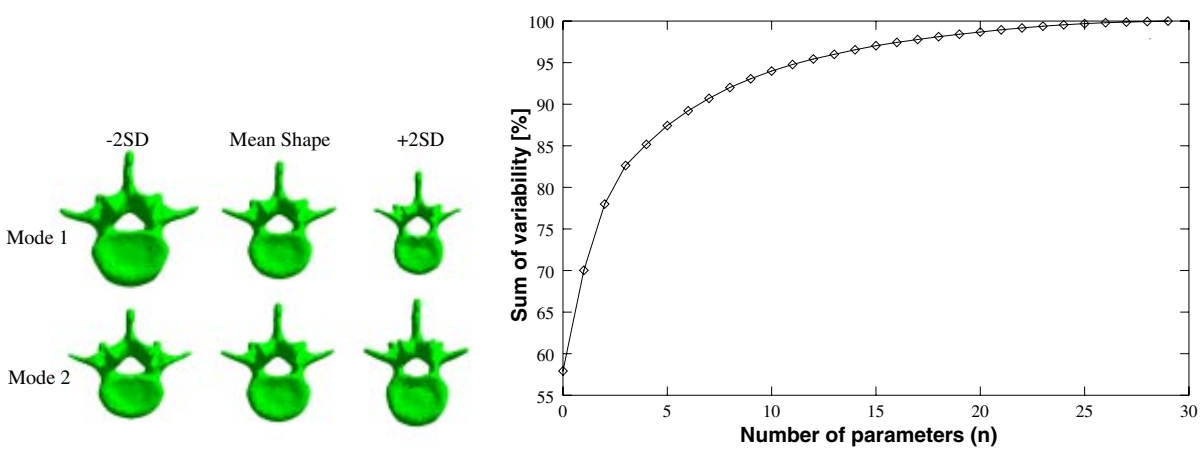

Fig. 4. Left: First two deformation modes of the shape model (axial view). Right: Captured variability of the statistical vertebra model as a function of the first $n$ eigenmodes in percent

is quite good although only very limited shape information was available (only few points in a restricted area were palpated). For 5 experiments the mean RMS between the vertebra to be reconstructed and the final model was $1.2 \mathrm{~mm}$.

\subsection{D Matching}

Experiments based on pure 2D data were performed using 2 orthogonal simulated $\mathrm{X}$-ray views based on 10 different CT-models that were not contained in the population used to build the model. In each simulated projection 400 randomly chosen contour points were maintained thus taking into account the fact that in practice not all contour points will be reliably detectable.

All datasets were then registered with the vertebrae shape model using all deformation modes. Fig [5] shows the shape model (mesh) after manual alignment 

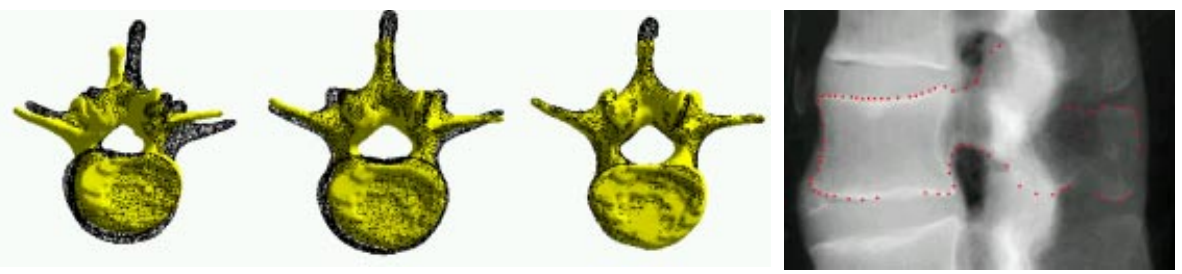

Fig. 5. Lumbar vertebra after manual alignment (left), after rigid registration (middle) and after non-rigid registration (right) Manually segmented contour points on a lateral $\mathrm{X}$-ray image of a cadaver spine; segmented points are enlarged for visualization) (most right)

(a), after rigid (b) and after non rigid (c) registration together with the CT-model from which the projections used for this experiment were generated. The final average RMS between the deformed shape model and the underlying CT-model (reference) was $0.62 \mathrm{~mm}$. The average overlap between the deformed shape model and the underlying CT-model was 91.12 percent.

Another experiment was performed using real data. A cadaveric lumbar spine was attached to a computer controlled turn-table and imaged twice for orthogonal turntable-angles using a prototype of an interventional X-ray imaging system equipped with a digital X-ray detector Prior to the experiment a CT-scan of the cadaver was acquired (voxel size: $0.27 \times 0.27 \times 1.0 \mathrm{~mm}^{3}$ ). The vertebra used for the experiment (L2) was segmented manually for later evaluation. L2 was segmented likewise manually in the two orthogonal X-ray images, see Fig. 5 for the lateral view. Subsequently the extracted contours were registered with the vertebra shape model using all deformation modes. The final average RMS between the deformed shape model and the CT-model was $1.27 \mathrm{~mm}$. The poorer result with respect to the experiment carried out with simulated projective data might be due to the fact that the cadaver spine belongs to a 80 year old specimen; the vertebrae show heavy degenerative changes. The computed shape model was based on a younger population, thus such shape variations occurring in older specimen are not gathered by the model.

Hybrid matching. Subsection 3 shows good reconstruction results based on relatively few scattered point data acquired in a highly restricted surface area. However, due to insufficient geometrical constraints ( depending on the shape and on the spatial distribution of the acquired points ), it might be mandatory to provide the correct pose parameters prior to the non rigid matching. In ea01a] authors report that the intra-operative rigid registration of a point cloud that has been acquired on a restricted dorsal surface area of a vertebra with a pre-operative acquired CT-model, can lead to unstable results (rotational uncertainty around the transversal axis) due to insufficient geometrical constraints in the accessible surface area of the vertebra. It is interesting to investigate if an additional X-ray image could provide sufficient extra information to suffi- 
ciently constrain the solution. This results in combining the methods proposed in the previous subsections, constituting a hybrid registration approach relying on both $2 \mathrm{D}$ projective and $3 \mathrm{D}$ point data. This is not only interesting for rigid registration but also for the more difficult problem of non rigid registration. An experiment was carried out using approximately 70 data points acquired on a CT based model in a small area accessible during spine surgery. Subsequently the shape model was fitted to both data - the 3D points and the segmented contours in the X-ray image - simultaneously. The final average RMS between the deformed shape model and the underlying CT-model (reference) was 0.68 $\mathrm{mm}$. This compares well with the results for pure $2 \mathrm{D} / 3 \mathrm{D}$ registration using two orthogonal X-ray views presented in the previous subsection.

\section{Discussion}

Obviously the presented approach can only be applied to healthy organs and shape pathologies that can be captured by statistical analysis of a population. Fractured organs cannot be modeled. However, various interventions could benefit from such methods. In the case of reconstruction of a torn cruciate ligament for instance, there is no pathologic shape variation of the adjacent bones (tibia, femur). Considering pedicle screw placement for spine instrumentation in the case of a vertebra compression fracture for instance, the shape of the vertebrae the screws are attached to (which are adjacent to the fractured one), is not pathologic; at least there is no pathologic shape variation associated to the reason for the surgery - the fracture. The possible benefits range from reduced radiation dose delivered to the patient, over decreased intervention time and overall inpatient time to the fact that the development of CAS systems with a more favorable cost/benefit ratio would be facilitated. This would help increasing the number of cases where sophisticated CAS technology is applicable and affordable.

\section{Acknowledgments}

The Philips research laboratories in Hamburg, the Helmholtz Institut in Aachen and Johns Hopkins Medical School in Baltimore are gratefully acknowledged for providing CT data. Financial support from the Region Rhone-Alpes and the european projects IGOSII, CRIGOS, MI3 is also gratefully acknowledged.

\section{References}

BM92. P.J. Besl and N.D. McKay. A method for registration of 3-D shapes. IEEE Transactions on Pattern Analysis and Machine Intelligence, 14(2):239-256, 1992.

ea99. J. Lotjonen et al. Model extraction from magnetic resonance volume data using the deformable pyramid. Mecial Image Analysis, 3(4):387-406, 1999. 
ea00. J. Montagnat et al. Surface simplex meshes for 3d medical image segmentation. In ICRA, 2000.

ea01a. A.F. Frangi et al. Automatic $3 \mathrm{~d}$ asm construction via atlas-based landmarking and volumetric elastic registration. In Proc. Information Processing in Medical imaging (IPMI'01), pages 78-91, 2001.

ea01b. C. Huberson et al. Surgical navigation for spine: Ct virtual imagery versus virtual fluoroscopy about 223 pedicle screws, in 88 patients. In CAOSUSA, pages 203-205, 2001.

FL98. M. Fleute and Stephane Lavallee. Building a Complete Surface Model from Sparse Data Using Statistical Shape Models: Application to Computer Assisted Knee Surgery. In W. M. Wells, A. Colchester, and S. Delp, editors, Medical Image Computing and Computer-Assisted Intervention-MICCAI'98, pages 880-887. Springer Verlag, October 1998.

FL99. M. Fleute and S. Lavallee. Nonrigid 3-D/2-D Registration of Images Using Statistical Models. In MICCAI'99, pages 138-147, 1999.

PFTV92. W. H. Press, B. P. Flannery, S. A. Teukolsky, and W. T. Vetterling. Numerical Recipes in $C$ : The Art of Scientific Computing. Cambridge University Press, Cambridge, England, second edition, 1992.

Pic97. R. Pichumani. Construction of a Three-dimensional Geometric Model for Segmentation and Visualization of Cervical Spine Images. PhD thesis, Stanford University School of Medicine, 1997.

SL96. R. Szeliski and S. Lavallee. Matching 3-D anatomical surfaces with nonrigid deformations using octree-splines. Int. J. of Computer Vision (IJCV), (18)(2):171-186, 1996.

SP86. T.W. Sederberg and S.R. Parry. Free-form deformations of solid geometric models. Computer Graphics (SIGGRAPH'86), 20(4):151-160, 1986.

Q1. What is the most important original contribution of the paper? This paper presents an integrated approach to the problem of intra-operative reconstruction of $3 \mathrm{D}$ anatomical surfaces. The method allows to use different intra-operatively available sparse shape and image data of different dimension to construct anatomical surfaces based on statistical shape models. A new approach for the automatic shape extraction from CT images necessary for the computation of the statistical model is also presented.

\section{Q2. What is the clinical relevance of the work presented?}

2. applied science ready for clinical inputs

5. cadaveric or animal studies were used in this work

\section{Q3. What is the most closely related work by other groups and how does your work differ?}

In [1] authors use biplanar X-ray images to reconstruct the 3D shape of scoliotic vertebrae. Our work differs by the fact that it allows to use different data sources simultaneously (scattered point data, ultrasound, X-ray images, etc). Further it is not explained in [1] how the vertebrae were segmented from the CT images and how they are aligned to compute the statistical model. This is a complex task especially when considering large databases which are necessary for a well performing resulting statistical model. Our work presents a highly automated approach for this task. 
Q4. Specify if this paper presents an extension of or close relates to some of your previously published work and state precisely the difference.

Our work presents an extension to [2] and [3] and [4]. The approach presented in this paper allows to use $3 \mathrm{D}$ data ([2]) and $2 \mathrm{D}$ data ([3]) simultaneously to reconstruct anatomical surfaces: The idea for the automatic shape extraction method has been presented in [4] but no matching results with real data were provided.

Q5. Specify the thematic categories and characterize your work best. Computer Assisted Surgery, Medical Imaging, Data Fusion, X-Ray images, Scattered Point Data, Shape Reconstruction, Shape Analysis, Deformable Model, Statistical Shape Model, Model Based Segmentation, Non-Rigid 3D/3D Registration, Non-Rigid 3D/2D Registration

\section{Q6. References, if any, for answering Q1 to Q5.}

1. S. Benameur, M. Mignotte, S. Parent, H. Labelle, W. Skalli, J. De Guise. 3D Biplanar reconstruction of scoliotic vertebrae using statistical models. International Conference on Computer Vision and Pattern Recognition, CVPR' 2001, Kauai Marriott, Hawaii, USA, Vol. 2, pages 577-582, December 2001.

2. M. Fleute, S. Lavallée. Building a Complete Surface Model from Sparse Data Using Statistical Shape Models: Application to Computer Assisted Knee Surgery. Medical Image Computing and Computer-Assisted InterventionMICCAI'98. Boston, USA, 1998.

3. M. Fleute, S. Lavallée. Nonrigid 3D/2D registration of Images using statistical models. MICCAI'99, Cambridge.

4. M. Fleute, L.Desbat, R.Martin, S. Lavallée, M. Defrise, X. Liu, R. Taylor. Statistical model registration for a C-arm CT system. IEEE Medical Imaging Conference 2001, San Diego, USA. 